\title{
A simple model for the prediction of yearly energy yields for grid- connected PV systems starting from monthly meteorological data
}

\author{
J. Muñoz , O. Perpiñán
}

\begin{abstract}
A B S T R A C T
This paper presents a simple model, called Clear-cloudy sky, which estimates yearly energy yields for PV systems starting from the twelve monthly values of global horizontal solar irradiation, diffuse fraction, Linke turbidity and minimum and maximum ambient temperatures. The proposed model has been included in an online and free-software simulator of PV systems, called SISIFO, which has been used to analyse the performance of the model in comparison with other synthetic models using as reference the typical meteorological years (TIMY3) of more than two hundred Class I stations belonging to the NREL American National Solar Radiation database. The results of this comparison show that the model provides yearly predictions on PV system performance parameters that have low bias and uncertainty with respect to the same figures obtained with the original TMY3 hourly time series.
\end{abstract}

\section{Introduction}

The prediction of yearly energy yield for a PV system is required in the early design stages of a project in order to estimate its technical performance and to analyse economic as well financing aspects. For this task, PV system designers and planners usually rely on public-domain or commercial software simulation tools with varying capabilities [1]. These simulation tools normally require, as input data, time series of irradiance and temperature coming from a variety of solar information resources. For example: monitoring of PV plants, ground-based meteorological stations, satellite data [2], or typical meteorological years (TMY). Besides, when these time series are not available for a particular site, some software tools, e.g. Meteonorm [3], may generate them using synthetic methods starting from a reduced set of meteorological information.

This paper presents a simple model, called Clear-Cloudy sky, which estimates yearly energy yields for PV systems starting from the twelve monthly values of global horizontal solar irradiation, diffuse fraction, Linke turbidity and minimum and maximum ambient temperatures. Using this meteorological information, the model generates time series of horizontal irradiances assuming an alternation of clear and fully cloudy days.
The performance of the model has been compared with other synthetic models using as reference TMY3 hourly time series of 217 Class I meteorological stations belonging to the NREL American National Solar Radiation database [4]. For the purposes of such comparison, the required monthly data to feed the models has been obtained from the TMY3 series themselves, but this information is usually available from public domain databases for any site in the world. For example, PVGIS [5] and NASA SSE [6] provide either this monthly data or other related information that allows its calculation.

The comparison has involved the simulation of a standard gridconnected PV system with three different PV generators (one static and two sun-trackers) using as inputs, first, TMY3 hourly data and, second, the time series generated by the synthetic models. The results of this simulation exercise show that the new model provides yearly predictions for PV system performance parameters that have low bias and uncertainty with respect to the same figures obtained with the original TMY3 time series.

Simulations have been carried out using an online [7] and freesoftware simulator of PV systems called SISIFO [8] [9]. The first version of SISIFO, which was released in October 2014 under GNU public license [10], allows the simulation of different types of gridconnected PV systems, such as large PV plants and building integrated installations. The GNU licence allows the users to download the kernel source code of SISIFO, written in the PHP programming language, which includes all the implemented models and algorithms.

This paper is structured as follows. Section 2 describes the proposed Clear-cloudy sky model and other synthetic generation models 
implemented in SISIFO. Section 3 describes the chain of model and algorithms implemented in the simulator and, finally, Section 4 compares the performance of the different synthetic models using as reference the above mentioned TMY3 hourly time series.

\section{Description of the proposed clear-cloudy sky model and other synthetic methods}

SISIFO accepts, as input data, time series of irradiance and ambient temperature with any time resolution (from seconds to hours) coming from multiple data resources: monitoring of PV plants, ground-based stations, satellite data, TMY, etc. But, if these time series are not available for a given location, SISIFO also offers the possibility of generating them starting from monthly meteorological data using three simple methods called Mean sky, Clear sky and the proposed Clear-Cloudy sky model, which are described in this section. Besides, two classic stochastic procedures proposed by Aguiar et al. [11,12] are also described, which are also compared with the previous simple methods.

\subsection{Mean sky model}

This model is an old and simple approach to the generation of time series of global horizontal irradiances, which requires as input the twelve monthly-averages of daily global irradiation, $G_{d m}(0)$. In this paper, the letter $G$ is used for global radiation, and $B$ and $D$ for its direct (beam) and diffuse components, respectively. If these radiation components have no subscript, they indicate instantaneous values (irradiances). Furthermore, the subscript ' $d m$ ' indicates a monthly-mean of daily values, and the zero between parentheses, $(0)$, the horizontal plane.

The mean sky model assumes that all the days of each month have the same daily horizontal global irradiation, which is equal to the average of that month, $G_{d m}(0)$. Time series of horizontal irradiances are obtained from $G_{d m}(0)$ applying the following algorithm.

1. Calculation of direct, $B_{d m}(0)$, and diffuse, $D_{d m}(0)$, irradiation components of $G_{d m}(0)$ :

$G_{d m}(0)=B_{d m}(0)+D_{d m}(0)$

$B_{d m}(0)=\left(1-K D_{d m}\right) G_{d m}(0)$

$D_{d m}(\mathbf{0})=K D_{d m} G_{d m}(0)$

Where $K D_{d m}$ is the diffuse fraction of global horizontal irradiation. This variable is a required input data, whose correct determination is of paramount importance to reduce the uncertainty of yearly energy predictions. If this information is not available, it can be estimated using experimental models that correlate the diffusefraction with the clearness-index [13-16].

2. Calculation of instantaneous values of diffuse $D(0)$, global $G(0)$, and direct $B(0)$, irradiances from monthly values using CollaresPereira and Rabl model [13]:

$$
\begin{aligned}
& D(0)=D_{d m}(0)\left(\frac{\pi}{T} \frac{\cos \omega-\cos \omega_{S}}{\sin \omega_{S}-\omega_{S} \cos \omega_{S}}\right) \\
& G(0)=G_{d m}(0)\left(\frac{\pi}{T} \frac{\cos \omega-\cos \omega_{S}}{\sin \omega_{S}-\omega_{S} \cos \omega_{S}}(a+b \cos \omega)\right) \\
& B(0)=G(0)-D(0)
\end{aligned}
$$

Where:

$$
\begin{aligned}
& a=0.409+0.5016 \sin \left(\omega_{S}-1.047\right) \\
& b=0.6609-0.4767 \sin \left(\omega_{S}-1.047\right)
\end{aligned}
$$

In the previous equations $\omega$ is the true solar time and $\omega_{S}$ the solar sunrise angle, which are given in radian, and $T$ is the length of the day. For example, $T$ must be set to 24 or 144 to calculate, respectively, hourly or 10 -min irradiance data.

The generated daily horizontal irradiance profiles are very similar for all the days of the month (see Fig. 1-a) and they only slightly differ in the peak of irradiance at midday, which decreases from one day to the next when the length of the day increases, and vice versa, to keep the same area under the curve.

This model preserves the input monthly mean value of global irradiation $\left(G_{d m}(0)\right)$ and diffuse fraction $\left(K D_{d m}\right)$, but it does not reproduce the real behaviour of the real sky, which alternates between clear days and cloudy days. However, despite its simplicity, it provides very good estimations of the radiation collected by static PV generators and slightly overestimates the radiation collected by sun-tracking planes.

\subsection{Clear sky model}

This model generates time series of irradiance that alternates between clear days and days with negligible (zero) solar radiation

(a)

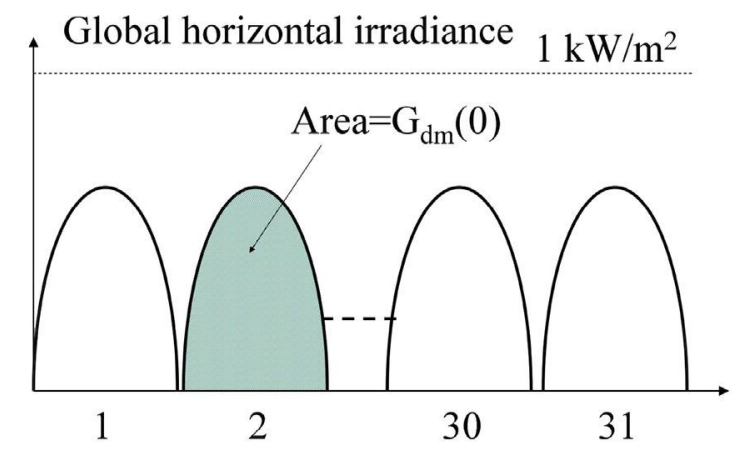

(b)

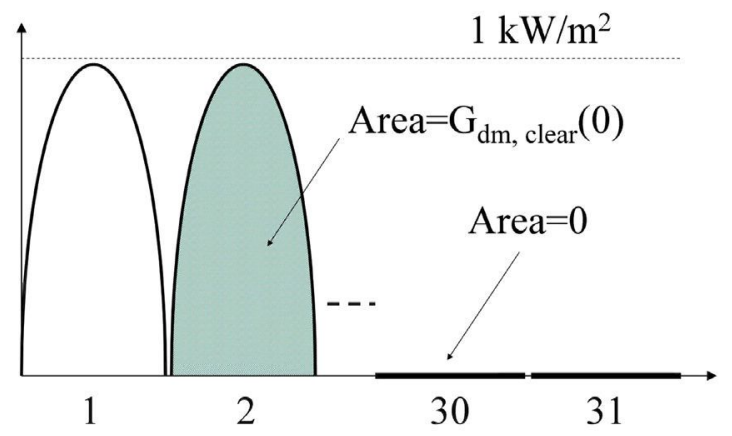

(c)

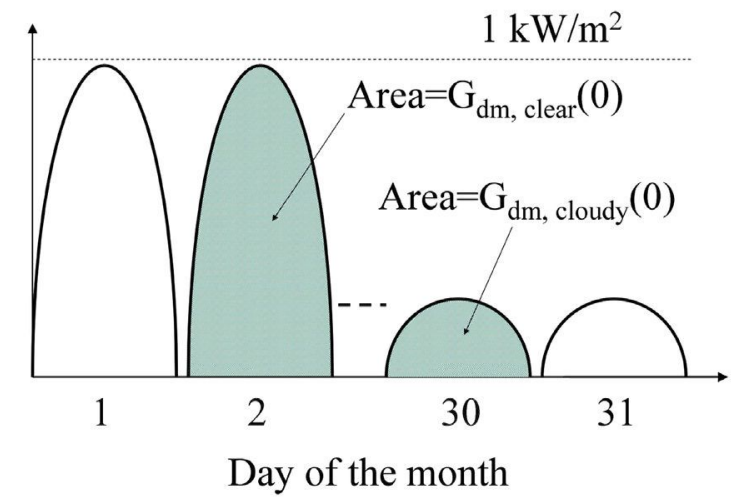

Fig. 1. Simple synthetic generation models implemented in SISIFO. (a) Mean sky. (b) Clear sky. (c) Clear-cloudy sky. 
(see Fig. 1-b). Direct and diffuse irradiances for clear days are calculated using the clear-sky model of the European Solar Radiation Atlas (ESRA) [18], which requires as inputs the altitude of the site and the Linke turbidity.

The model preserves the monthly mean value of global irradiation, $G_{d m}(0)$, ensuring that the monthly fraction of clear days in the generated time series is equal to the so-called clear-sky index, $K_{C}$, which is defined as the ratio:

$K_{C}=\frac{G_{d m}(0)}{G_{d m, \text { clear }}(0)}$

Where $G_{d m}(0)$ is the horizontal monthly mean daily irradiation of the site and $G_{d m, c l e a r}(0)$ is the same quantity but obtained assuming that all the days of the month are clear.

Nevertheless, the model does not preserve the diffuse fraction, which is normally underestimated to a great extent. It is shown below that this model largely overestimates the collected energy on the plane of PV generators, in particular with solar trackers. Therefore, its use is not recommended for energy yield predictions, although it may be useful for other purposes. For example, exploring PV system performance under high-radiation conditions.

\subsection{Clear-cloudy sky model}

This model generates time series of horizontal irradiances assuming an ideal alternation of clear and fully cloudy days (see Fig. 1-c) combining the characteristics of the above described models. On the one hand, direct and diffuse horizontal irradiances of the clear days are calculated using the ESRA model [18] using as inputs the altitude of the site and Linke turbidity. On the other hand, horizontal direct irradiances of cloudy days are assumed to be null and diffuse horizontal irradiances are determined according to Collares-Pereira and Rabl model [13] using Eq. (2). The latter requires the estimation of the horizontal diffuse irradiation for cloudy days, $D_{d m, \text { cloudy }}(0)$, whose calculation is detailed below.

The proportions of clear and cloudy days are calculated to ensure two conditions. First, that the monthly-mean daily global horizontal irradiation of the synthetic time series matches the monthly input value, $G_{d m}(0)$. And, second, that monthly diffuse fraction of $G_{d m}(0)$ is preserved. In order to ensure both conditions, the following quantities are calculated:

1. Direct, $B_{d m}(0)$, and diffuse, $D_{d m}(0)$, irradiation components of $G_{d m}(0)$ using Eq. (1).

2. Global, direct and diffuse irradiances using the ESRA model [18], and their integration to obtain monthly mean values of global, beam and diffuse daily irradiations for clear days:

$G_{d m, \text { clear }}(0)=B_{d m, \text { clear }}(0)+D_{d m, \text { clear }}(0)$

3. Monthly fraction of clear days, $K_{\mathrm{C}}$, which must ensure that direct irradiation of the clear days matches the direct irradiation of the month (since, by model assumption, the direct radiation during cloudy days is null). Hence:

$K_{C}=\frac{B_{d m}(0)}{B_{d m, \text { clear }}(0)}$

4. Diffuse irradiation for cloudy days, $D_{d m \text {, cloudy }}(0)$, which must preserve the monthly diffuse fraction of the month. This requires that the weighted arithmetic mean of diffuse irradiations for clear and cloudy days is equal to the monthly diffuse irradiation, $D_{d m}(0)$. Therefore:
$D_{d m}(\mathbf{0})=K_{C} \cdot D_{d m, \text { clear }}(\mathbf{0})+\left(1-K_{C}\right) \cdot D_{d m, \text { cloudy }}(0)$

Where $\left(1-K_{C}\right)$ is the monthly fraction of cloudy days. From the previous equation, the required diffuse irradiation for cloudy days, $D_{d m, \text { cloudy }}(0)$, is calculated as:

$D_{d m, \text { cloudy }}(0)=\frac{D_{d m}(0)-K_{C} \cdot D_{d m, \text { clear }}(0)}{1-K_{C}}$

5. Finally, it is easy to verify that global irradiation of the daily sequence is the same than the original value:

$$
\begin{aligned}
G_{d m}(0) & =K_{C} \cdot G_{d m, \text { clear }}(0)+\left(1-K_{C}\right) \cdot G_{d m, \text { loudy }}(0) \\
& =B_{d m}(0)+D_{d m}(0)
\end{aligned}
$$

The previous algorithm is applied for each month of the year to obtain twelve time series of horizontal irradiances whose daily profiles alternate between clear and cloudy days, as displayed in Fig. 1-c. In practise, two simulations are carried out. The former assuming that all days are clear and the latter assuming all days are fully cloudy. Finally, as the PV system operates a fraction of the time under clear days $\left(K_{C}\right)$ and the complementary fraction under cloudy days $\left(1-K_{C}\right)$, the calculation of any monthly energy parameter for the PV system, $E_{\text {month }}$, is calculated as the weighted arithmetic mean of both extreme operations:

$E_{\text {month }}=K_{C} \cdot E_{\text {month,clear }}+\left(1-K_{C}\right) \cdot E_{\text {month,cloudy }}$

Where $E_{\text {month, clear }}$ is the calculated monthly energy assuming that all the days of the month are clear, and $E_{\text {month, cloudy }}$ is the same quantity but assuming that all the days of the month are cloudy.

\subsection{Stochastic models}

The performance of the previous three simple models has been compared with two stochastic models proposed by Aguiar et al., which are based on the use of a library of Markov transition matrices [11] and autoregressive processes [12]. These models are called in this paper Aguiar1 and Aguiar2, respectively.

Aguiar1 model generates a daily sequence of clearness indices using as only input the monthly clearness index, which is calculated from the ratio between the input monthly mean value of global horizontal irradiation, $G_{d m}(0)$, and the extraterrestrial irradiation for that month. First, the daily sequence of clearness indices is converted into a daily sequence of horizontal daily global irradiation. Next, each daily global horizontal irradiation is separated into direct and diffuse components using daily diffuse-fraction calculated with experimental clearness-index correlations [13,15-17], and applying Eq. (1). Finally, the instantaneous values of direct and diffuse irradiances are calculated using Eq. (2).

Aguiar2 model starts from the daily sequence of clearness indices generated by Aguiar1 model and synthesises hourly sequences of clearness indices, which are later converted to hourly global horizontal irradiances. Next, these hourly global irradiances are separated into direct and diffuse components. For example, using the experimental models described by Erbs [15] or OrgillHollands [19].

\section{Models and algorithms implemented in SISIFO}

This section summarises the models implemented in SISIFO for simulating the behaviour of grid-connected PV systems. For conciseness, the details of most of the models are not discussed here. The interested reader may consult the original referenced 
publications or the source code of the program, where equations and algorithms are written and widely commented.

Assuming that the required time series of horizontal irradiances (global, direct and diffuse) are already available, the sequence of calculations involves two main steps. The first one is determining the operating conditions of the PV generator, i.e., the global irradiance incident on its surface, after discounting the corresponding energy losses, $G$, and cell temperature, $T_{C}$.

The second step is calculating the chain of power conversions from the PV generator to the output power of the low voltage/ medium voltage (LV/MV) transformer, which is finally injected into the electricity grid.

\subsection{Determination of the operating conditions}

The sequence of calculations implemented in SISIFO to determine the global irradiance on the PV generator plane is the following:

1. Determination of the positions of the Sun and the PV generator surface, and the angle of incidence [20]. SISIFO offers the possibility of selecting amongst three static structures and six suntrackers with the option of backtracking [21].

2. Geometric calculation of self-shading area on the PV generator surface caused by neighbouring PV generators. If this shaded area is not null and the backtracking mode of operation has been selected, the tracker rotates on the selected axis to eliminate the shadow [21], which requires recalculating the angle-ofincidence of the Sun on the PV generator surface.

3. Calculation of the irradiance on the plane of the PV generator using isotropic or anisotropic, Hay [22] or Perez [23], diffuse radiation models. The simple isotropic approximation is offered just for completeness, since it is well- known that this model systematically underestimates the diffuse component on tilted planes (see, e.g., reference [24]).

4. Finally, the previously calculated irradiance value is corrected taking into account the impact of the angle-of-incidence and soiling [25], self-shading of PV generators [26] and solar spectrum [27], to obtain the final global irradiance, $G$.

The second operating condition, cell temperature $T_{C}$, is calculated from the ambient temperature, $T_{A}$, using the well-known equation based on the nominal operation cell temperature, NOCT:

$T_{C}=T_{A}+0.9 \frac{N O C T-20}{800} G$

Where $T_{A}$ and NOCT are given in ${ }^{\circ} \mathrm{C}$ and $G$ is in $\mathrm{W} \cdot \mathrm{m}^{-2}$. The factor 0.9 is an experimental correction factor, based on experimental evidence, which averages the cooling effect of wind speed on openback mounted PV modules in large PV plants.

The diurnal variation of ambient temperature, which is considered to be the same for all the days of each month, is generated using a cosine-type model that makes an interpolation between the monthly average of the minimum and maximum daily ambient temperatures assuming that minimum occurs at sunrise and the maximum two hours after midday [20]. For the purposes of comparing the model with TMY3 series, these monthly mean values for minimum and maximum ambient temperatures have been obtained from TMY3 data.

\subsection{Determination of the power injected in the grid}

Once the operating conditions, $G$ and $T_{\mathcal{C}}$, have been determined, SISIFO calculates the chain of power conversions from the PV generator to the output power of the LV/MV transformer, POUT, which is finally injected into the electricity grid.

Fig. 2 shows the general configuration of the simulated gridconnected PV system, which is made up of a PV generator, an inverter, and a low voltage/medium voltage (LV/MV) transformer, whose output powers are, respectively, $P_{D C}, P_{A C}$, and $P_{O U T}$.

There is a wide variety of models proposed in the literature for describing the behaviour of PV generators and Balance-Of-System (BOS) components. Looking for the experimental validation of the predicted behaviour of the PV system, the selected models for SISIFO are uniquely based on parameters available in component datasheets, which may be guaranteed by manufacturers and verified by on-site testing campaigns [28] [29].

\subsubsection{PV generator model}

The maximum PV generator power, $P_{D C}$, is calculated using the following maximum power point model:

$P_{D C}=P^{*} \frac{G}{G^{*}}\left[1+\gamma\left(T_{C}-T_{C}^{*}\right)\right]\left[a+b \frac{G}{G^{*}}+c \ln \frac{G}{G^{*}}\right]$

Where $P^{*}$ is the maximum power of the PV generator under Standard Test Conditions (or STC, indicated here by the superscript '*' and defined by a normal irradiance of $G^{*}=1000 \mathrm{~W} / \mathrm{m}^{2}$, cell temperature $T_{\mathrm{C}}^{*}=25^{\circ} \mathrm{C}$, and AM1.5 spectrum). The value of $P^{*}$ is obtained either from manufacturer information (as the sum of the nominal power of the PV modules according to their nameplate or flashing reports) or from experimental measurements [28]. Gamma, $\gamma$, is the coefficient of variation of power with temperature, in ${ }^{\circ} \mathrm{C}^{-1}$. And $a, b$, and $c$ are three parameters that must be fitted for each PV module to model the variation of the PV power efficiency with the level of irradiance [30].

\subsubsection{Inverter model}

Balance-of-system components are described by general power efficiency models that are also based on manufacturer datasheet information. The power efficiency of the inverter, $\eta_{\text {I }}$, is modelled using the following equation [31]:

$$
\eta_{I}=\frac{P_{A C}}{P_{D C}}=\frac{p_{a c}}{p_{a c}+\left(k_{0}+k_{1} p_{a c}+k_{2} p_{a c}^{2}\right)}
$$

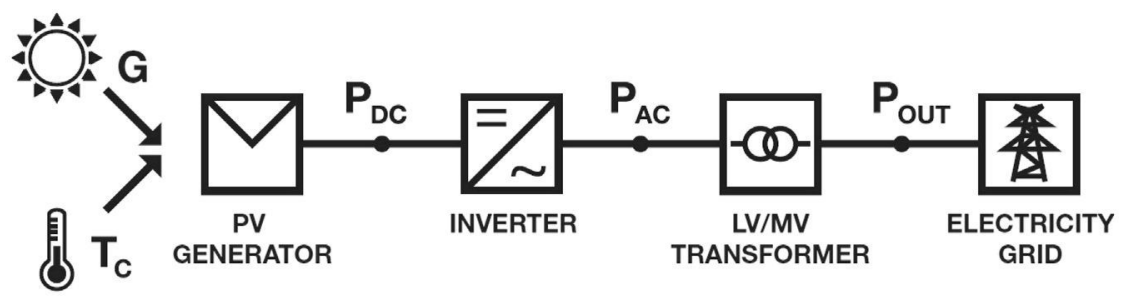

Fig. 2. Configuration of the simulated grid-connected PV system, which is made up of a PV generator, inverter, and LV/MV transformer. 
Where $p_{a c}=P_{A C} P_{I}, P_{I}$ being the nominal power of the inverter and $P_{A C}$ its output $A C$ power, which can be determined from $P_{D C}$ (power at the inverter input) and parameters $k_{0}, k_{1}$ and $k_{2}$. These parameters are fitted either from the power efficiency curve provided by the inverter manufacturer or from experimental on-site measurements [29].

\subsubsection{Transformer model}

Similarly, the power efficiency of the LV/MV transformer, $\eta_{T}$, is calculated as [32]:

$\eta_{T}=\frac{P_{\text {OUT }}}{P_{\text {AC }}}=\frac{P_{\text {OUT }}}{P_{\text {OUT }}+P_{\text {Core }}+P_{C u}}$

where $P_{\text {out }}$ is the transformer output power, $P_{\text {Core }}$ are iron losses, and $P_{C u}$ are copper losses, which are calculated as:

$P_{C u}=P_{C u, n o m} \cdot\left(\frac{P_{\text {OUT }}}{P_{T}}\right)^{2}$

Where $P_{C u \text { nom }}$ are the copper losses when the transformer operates at its nominal output power, $P_{T}$. The required model parameters, $P_{\text {Core }}$ and $P_{C u, n o m}$ are obtained from manufacturer datasheet.

Finally, the output power injected into the grid, $P_{O U T}$, is calculated in each instant using the previous equations as:

$P_{\text {OUT }}=P_{A C} \eta_{T}=P_{D C} \eta_{I} \eta_{T}$

\section{Comparison between the synthetic models and TMY3 time series}

The performance of the synthetic models has been compared using as reference the typical meteorological years of 217 Class-I stations of the USA NSRDB database (TMY3) [4], which are nearly uniformly distributed across the country covering different latitudes, altitudes and climate regions (see Fig. 3 ).

The results of this comparison are presented in two parts. The first one, which is discussed in Section 4.1, treats on the calculation of yearly solar irradiation on the tilted plane of PV generators starting from hourly time series of horizontal irradiances. This first part compares the performance of the five synthetic models, described in Section 2, and TMY3 data.

The second part of the simulation exercise, which is described in Section 4.2, deals with the conversion from the calculated

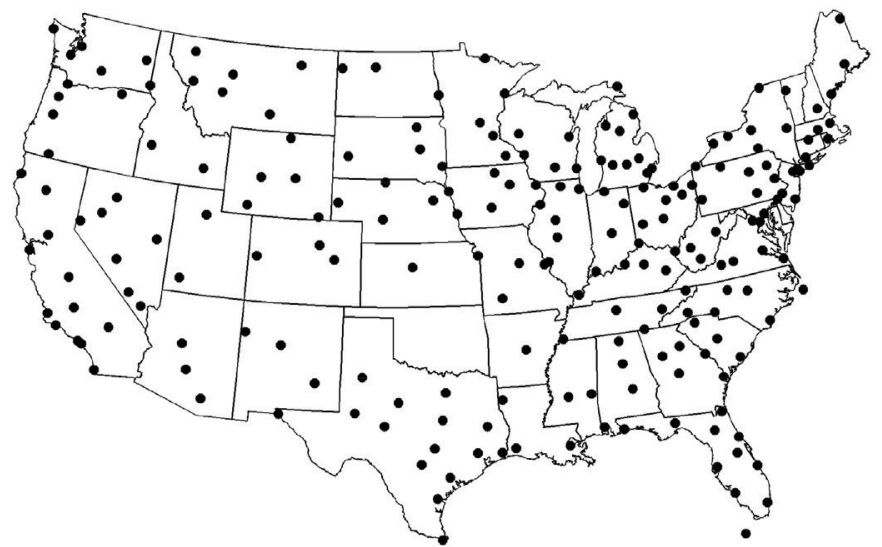

Fig. 3. Geographical distribution of the 217 Class I stations of the NSRDB database used as reference in the simulations. irradiances on the PV generator plane into time series of electric power, which are integrated to compare yearly values of PV system losses and final energy yields. This second part only compares the new model, Clear-cloudy sky, with TMY3 data.

\subsection{Yearly irradiation on the PV array plane}

For each station, the plane-of-array (POA) irradiance on three tilted planes has been calculated and integrated to obtain the POA yearly irradiation. The selected planes are a static structure oriented to the Equator and tilted the latitude, one-axis N-S horizontal tracker and two-axes tracker, which have been largely separated to neglect the impact of self-shading losses. It has been assumed that both trackers, ideally, have not any mechanical limitation to reach their optimum position, which collects the maximum solar irradiance for each instant.

Furthermore, the monthly mean values of the TMY3 time series have been calculated to feed the model with the exception of Linke turbidity, which has been estimated from other TMY3 data (atmospheric water vapor and aerosol content) using the conversion function proposed by Ineichen [33].

The statistical indicators used to asses the performance of the synthetic model have been the relative Mean Bias Difference (MBD) and the relative root mean square difference (RMSD), which are defined as:

$$
\begin{gathered}
M B D=\frac{\frac{1}{N} \sum_{i=1}^{N}\left(M O D E L_{i}-T M Y\right)_{i}}{\frac{1}{N} \sum_{i=1}^{N} T M Y_{i}} \\
\text { RMSD }=\frac{\sqrt{\frac{1}{N} \sum_{i=1}^{N}\left(M O D E L_{i}-T M Y_{i}\right)^{2}}}{\frac{1}{N} \sum_{i=1}^{N} T M Y_{i}}
\end{gathered}
$$

Where $M O D E L_{i}$ and $T M Y_{i}$ are, respectively, the calculated values using the model and the original TMY3 series for ith station, being $N$ the number of stations $(N=217)$. The $M B D$ is used to indicate if the model underestimates or overestimates the predictions provided by TMY3 data. The RMSD represents the standard deviation of the differences between the model and TMY3 data.

Before comparing the performance of the models, it is worth mentioning that stochastic models, by their own nature, generate time series of global horizontal irradiances whose integrated yearly values are different from the original TMY3 data. To minimise this effect, stochastic series have been generated imposing as convergence criterion that synthetic monthly clearness indices differ less than $\pm 1 \%$ from TMY3 values. As result, in yearly terms, the generated stochastic series have an initial off-set $M B D= \pm 0.03 \%$ and a maximum $R M S D=0.4 \%$, while the simple models have not any initial deviation $(M B D=R M S D=0 \%)$.

Besides, the performance of stochastic methods depends on the model applied for separating the horizontal global radiation into its direct and diffuse components, which is a major source of uncertainty in the calculation of the irradiance on the PV array plane [24]. In this paper, Aguiar1 has been used in combination with CollaresPereira and Rabl model [13], and Aguiar2 with Erbs model [15].

Fig. 4 shows the scatter plots of the POA yearly solar irradiation calculated using the TMY3 time series, which is considered here the 'true' value, and the prediction provided by the five synthetic models. All the calculations have been performed assuming an anisotropic diffuse distribution (Hay [22]) and a constant ground reflectance equal 

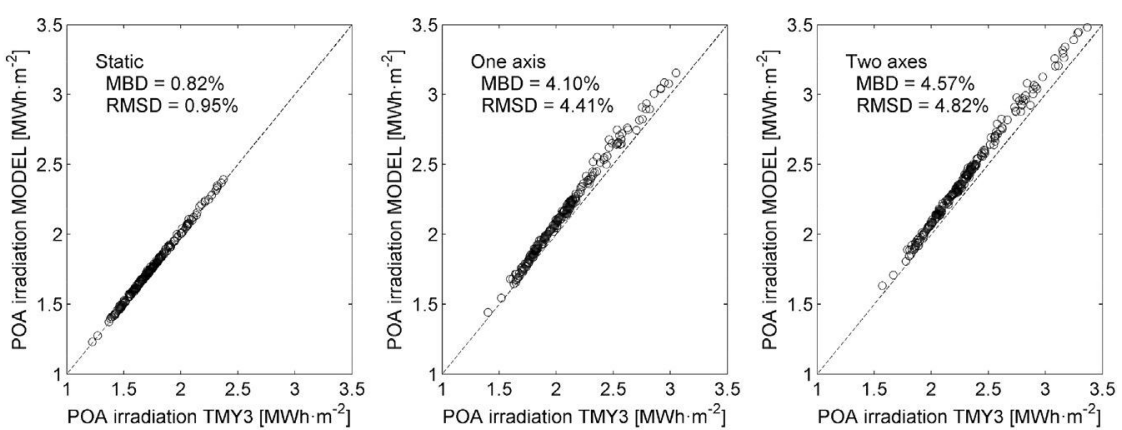

(a)
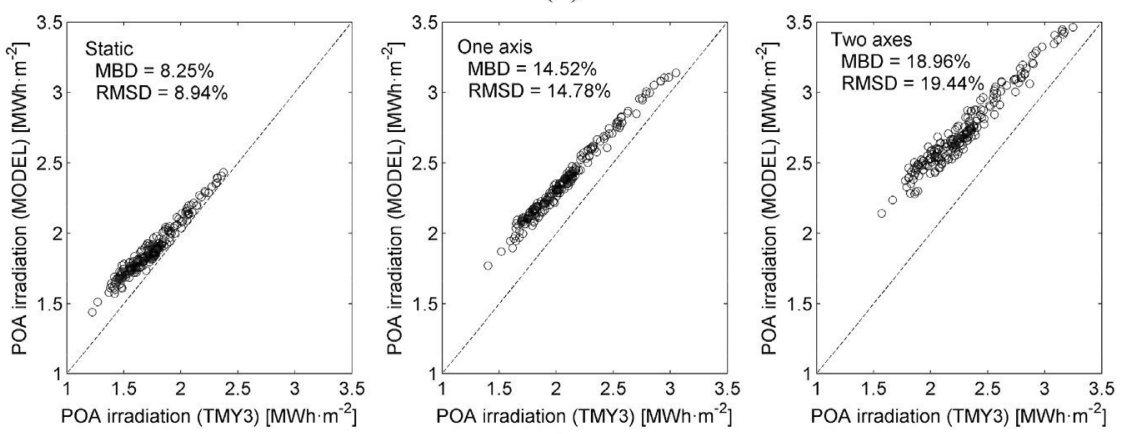

(b)
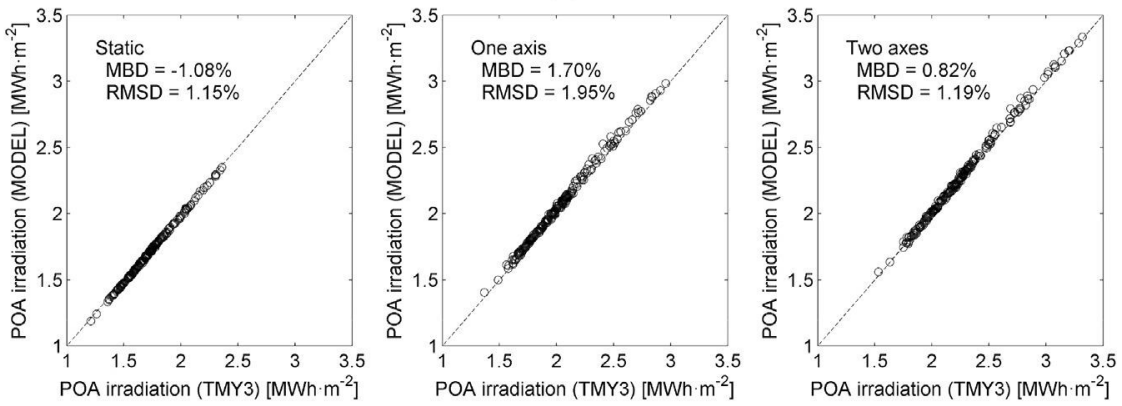

(c)
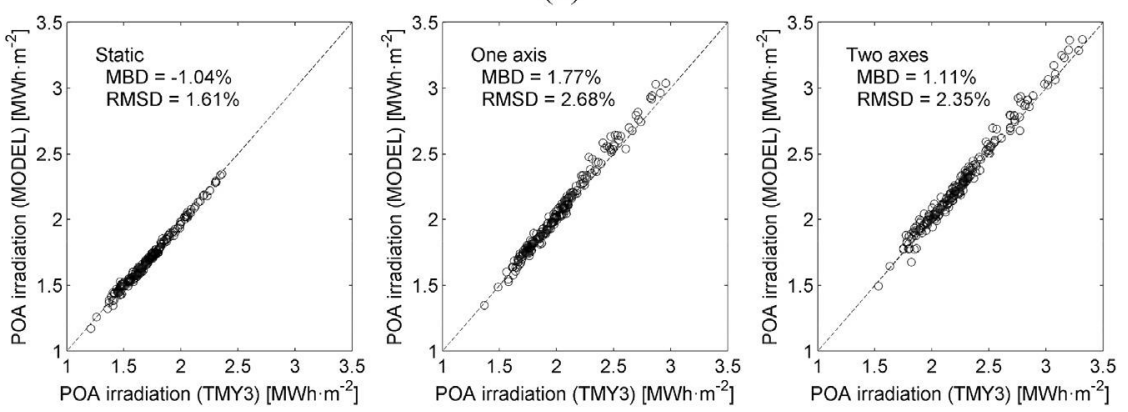

(d)
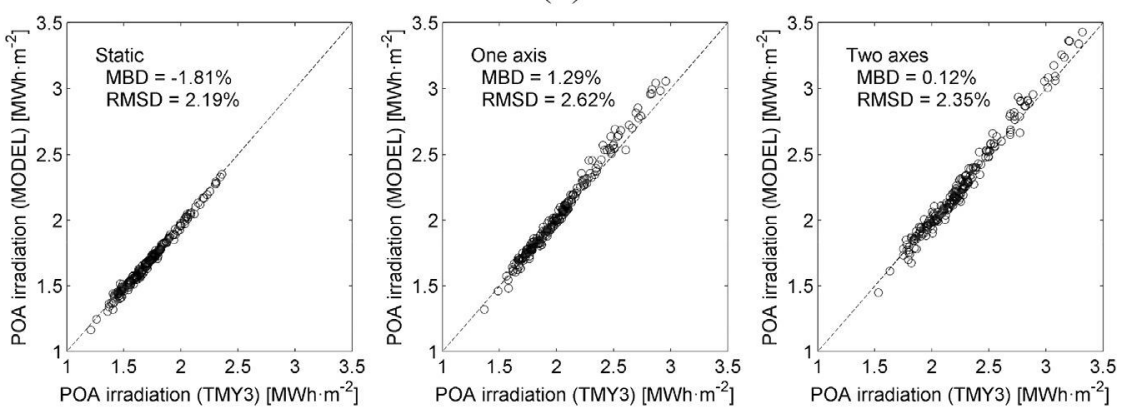

(e)

Fig. 4. Comparison between the calculated POA yearly solar irradiation using the TMY3 time series and the predicted value by the synthetic models. (a) Mean sky. (b) Clear sky. (c) Clear-cloudy sky. (d) Aguiar1. (e) Aguiar2. 
to 0.2 , which is the usual value when this parameter is unknown.

It can be observed that the proposed Clear-cloudy sky model (Fig. 4-c), is very consistent for the three simulated tilted planes and exhibits maximum $M B D$ and $R M S D$ within $\pm 2 \%$ in all the cases. Compared with the other synthetic models:

- The Mean sky model (Fig. 4-a) performs slightly better for the static PV generator, but it gets worse with both sun-trackers, reaching $M B D$ that exceeds $4 \%$ and $R M S D$ values around $5 \%$.

- The Clear sky model (Fig. 4-b), as expected, largely overestimates the yearly POA irradiation. Obviously, its use is discouraged for energy yield prediction but it can be still useful for other purposes, such us exploring the performance of the PV system under high radiation conditions.

- Aguiar1 (Fig. 4-d) and Aguiar2 (Fig. 4-e) models show a similar performance, but their RMSD is somewhat higher.

In summary, the Clear-Cloudy sky model substantially improves the predictions provided by the other simple models, Mean sky and Clear sky, and its performance is comparable with Aguiar1 and Aguiar2 stochastic generation methods. As additional feature, the new model requires lower computation time, which is an advantage for concurrent simulations, for example, in multi-user web applications. From now on, only the Clear-Cloudy sky model will be compared with TMY3 data in order to simplify the presentation.

In the previous simulations, the Clear-Cloudy sky model starts from the optimum values for the monthly averages, which are obtained from the original TMY3 time series. In practise, the model is fed with monthly meteorological data: global horizontal monthly irradiation, diffuse fraction and Linke turbidity [34,35], which are easy to find, or derived, from available data. For example, diffuse fraction is usually estimated using experimental cross-correlations with clearness index [13-16].

Table 1 shows the statistical indicators of the comparison between the POA yearly solar irradiation calculated with the TMY3 series and the prediction provided by the combination of the Clear-cloudy sky model and three monthly diffuse fraction correlations, which have been derived for locations in the U.S. [13,15]. It can be observed that the $M B D$ with sun-trackers decreases using the correlations of Collares and Page, but in most cases RMSD increases regarding the optimum values for diffuse fraction obtained from TMY3.

\subsection{PV system losses and final yields}

The second part of the simulation exercise deals with the conversion from the calculated irradiances on the PV generator plane into time series of electric power, which are integrated to compare yearly energy losses and final energy yields. The analysed energy losses are those caused by angle-of-incidence, cell temperature, inverter and transformer.

All the calculations have been performed assuming Hay anisotropic diffuse sky model [22], a ground reflectance equal to 0.2 , and

Table 1

Comparison between the statistical indicators for deviations between the calculated POA yearly solar irradiation using the TMY3 time series and the predicted value by the Clear-cloudy sky model using three experimental monthly diffuse fraction correlations and Hay model.

\begin{tabular}{|c|c|c|c|c|c|c|}
\hline \multirow{2}{*}{$\begin{array}{l}\text { Diffuse fraction } \\
\text { calculated using }\end{array}$} & \multicolumn{2}{|l|}{ Static } & \multicolumn{2}{|c|}{ One axis } & \multicolumn{2}{|c|}{ Two axes } \\
\hline & $\mathrm{MBD}, \%$ & RMSD, \% & $\mathrm{MBD}, \%$ & RMSD, \% & MBD, \% & RMSD, \% \\
\hline $\begin{array}{l}\text { TMY3 data } \\
\text { (optimum) }\end{array}$ & -1.08 & 1.15 & 1.70 & 1.95 & 0.82 & 1.19 \\
\hline Collares [13] & -1.40 & 2.01 & 0.61 & 1.65 & 0.26 & 2.19 \\
\hline Page [14] & -2.26 & 2.48 & 1.12 & 1.63 & 0.12 & 1.54 \\
\hline Erbs [15] & -1.16 & 1.64 & 2.64 & 2.90 & 2.16 & 2.73 \\
\hline
\end{tabular}

using as reference a standard 1 MWPV system whose characteristics and model parameters are given in Table 2.

Table 2

Characteristic and model parameters of the simulated PV system.

\begin{tabular}{ll}
\hline PV modules & $c-S i\left(\gamma=0.5 \% \mathrm{C}^{-1}\right)$ \\
& Negligible variation of PV efficiency with \\
& irradiance $(a=1, b=c=0)$ \\
& $N O C T=48^{\circ} \mathrm{C}$ \\
& Nominal power $=1 \mathrm{MW}_{\mathrm{P}}$ \\
PV generator & - Structures:Static and tilted the latitude \\
& - One axis horizontal N-S tracker \\
& - Two axes tracker \\
& Negligible self-shading, soiling and spectral effects. \\
Inverter & $P_{I}=1 \mathrm{MW}, k_{0}=0.01, k_{1}=0.002$ and $k_{2}=0.04$ \\
Transformer & $P_{T}=1 \mathrm{MW}, P_{\text {Core }}=1 \mathrm{~kW}, P_{C u}=10 \mathrm{~kW}$ \\
Wiring losses & Null \\
\hline
\end{tabular}

\subsubsection{Angle-of-incidence losses}

Angle-of-incidence losses have been estimated using Martin and Ruiz model [25]. This model includes the combined effects of the angle-of-incidence of Sun rays regarding the collected surface and soiling losses, which have been assumed to be null in the simulation exercise. In practise, the degree of soiling on the surface of the PV modules is not negligible and strongly depends on the particular characteristics of the site (see, e.g. Ref. [36]), which is a major source of uncertainty in the prediction of PV system energy yields.

Fig. 5 compares the estimated angle-of-incidence losses with the model regarding the predictions of TMY3 data. As expected, these losses decrease with sun-trackers, in particular with the two axes one, whose surface remains perpendicular to the Sun rays all the time. The average angle-of-incidence yearly energy losses calculated with TMY3 data are $3.19 \%, 1.74 \%$ and $1.21 \%$, respectively, for the static, one-axis and two-axis PV generators. It can be observed that the model overestimates incidence losses but, as their magnitude is relatively small (from $0.5 \%$ to $3.5 \%$ ), the impact on final energy yields is negligible.

\subsubsection{Cell temperature losses}

Cell temperature energy losses represent a major loss factor in the PV system. For all the simulated locations, see Fig. 6, the calculated yearly temperature losses with TMY3 data vary between around $0 \%$, for cold climates, and $15 \%$, for warm climates.

The Clear-cloudy sky model estimates these losses with a maximum RMSD around 5\% regarding TMY3 data (see Fig. 6), which does not introduce a substantial error in the calculation of final PV energy yields.

\subsubsection{Inverter and transformer losses}

Fig. 7 displays the yearly energy losses caused by the inverter which are nearly constant around 5\% for all the simulated cases. This occurs because the power efficiency curve of the selected inverter is nearly flat for medium and high output power levels, which is a characteristic of inverters with small self-consumption losses.

The Clear-cloudy sky model estimates these losses with deviations of around 5\% regarding the TMY3 reference. The model provides similar deviations for the LV/MV transformer losses, but their magnitude are so small, around $1 \%$, that they are not represented in the figure.

\subsubsection{Final energy yields}

As discussed above, the synthetic model overestimates some 

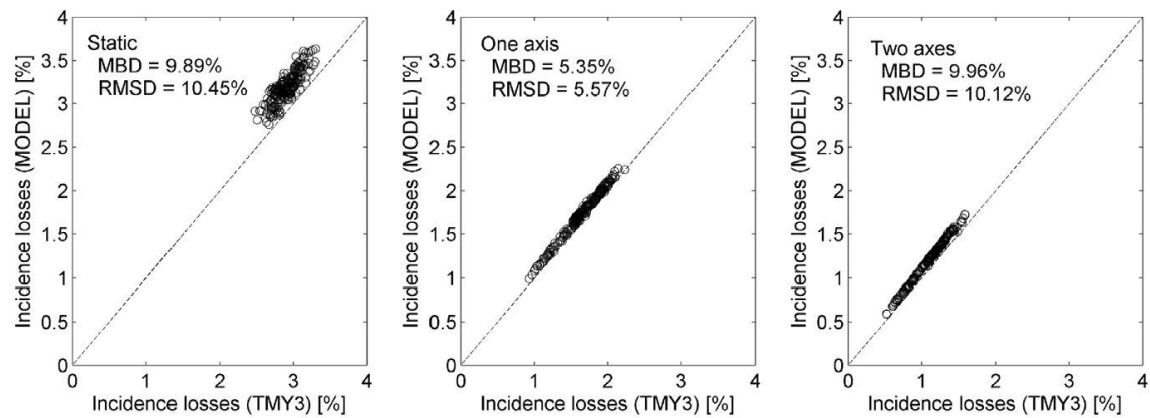

Fig. 5. Comparison between yearly energy losses, caused by angle-of-incidence, calculated using the TMY3 time series and the Clear-cloudy sky model.
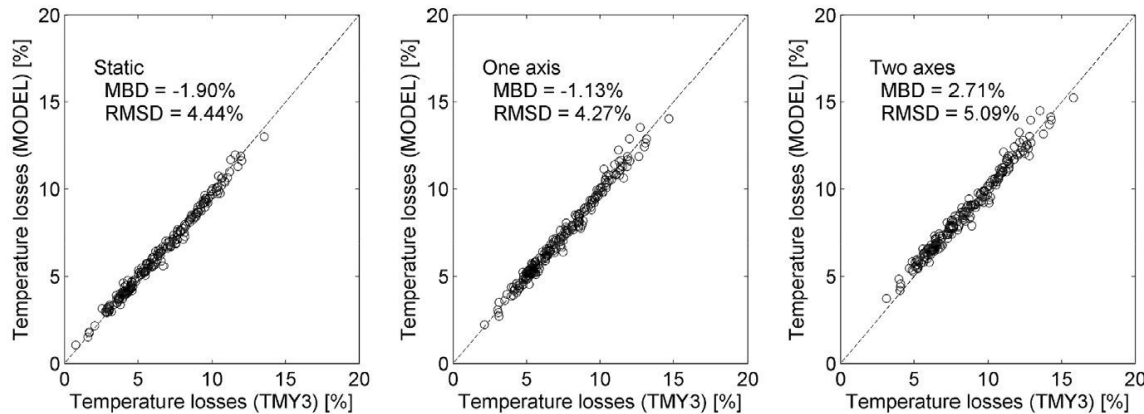

Fig. 6. Comparison between yearly energy losses, caused by cell temperature, calculated using the TMY3 time series and the Clear-cloudy sky model.
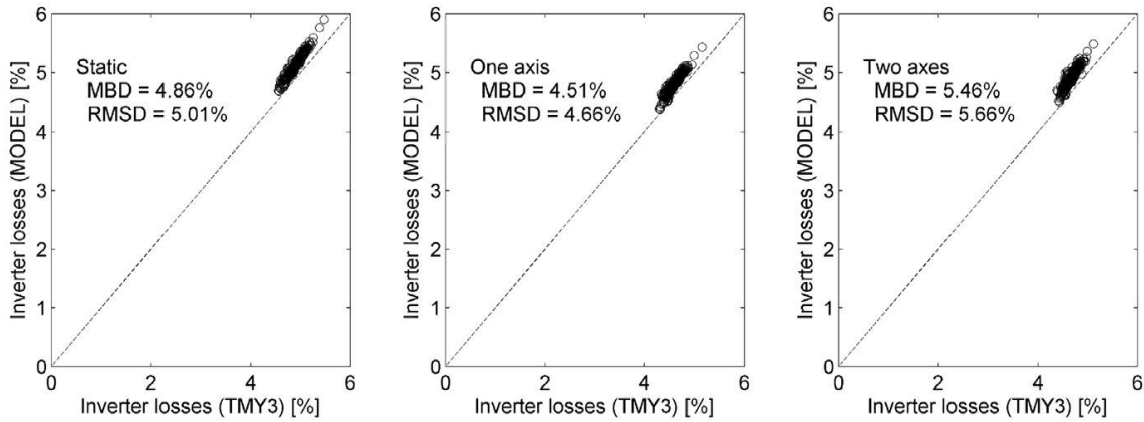

Fig. 7. Comparison between yearly energy losses, caused by the inverter, calculated using the TMY3 time series and the Clear-cloudy sky model.

yearly losses and underestimate others, but the combined effect on the estimation of final energy yields is relatively small (see Fig. 8). As result, the Clear-cloudy sky model provides predictions that are very similar to the results obtained with the original TMY3 time series, with maximum deviations that are lower than $\pm 2 \%$ in terms of both $M B D$ and RMSD.

\section{Conclusions}

A simple model, called Clear-Cloudy sky, has been presented in this paper, which allows the prediction of final yearly energy yields for PV systems starting from a reduced dataset of monthly meteorological information: global horizontal solar irradiation, diffuse
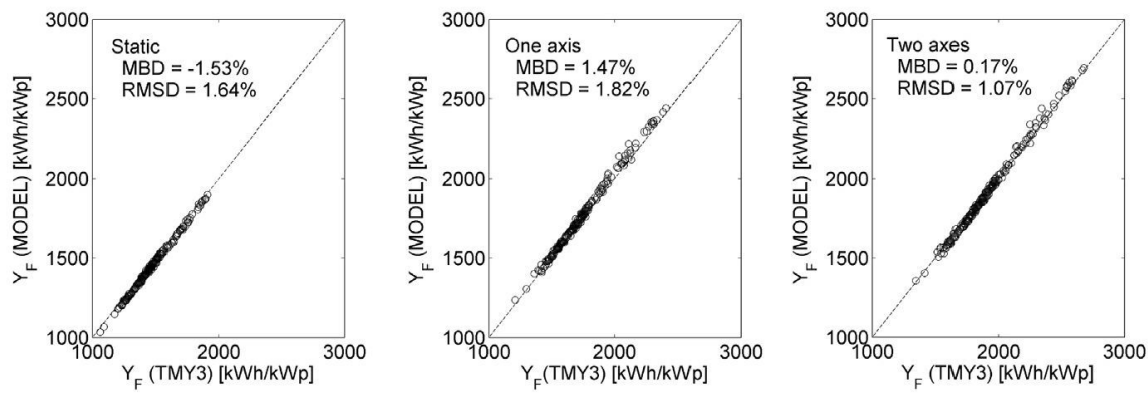

Fig. 8. Comparison between final energy yields $\left(\mathrm{Y}_{\mathrm{F}}\right)$ calculated using the TMY3 time series and the Clear-cloudy sky model. 
fraction, Linke turbidity, and minimum and maximum ambient temperatures.

The model has been included in an online and free-software simulator of PV systems, called SISIFO, which has been employed to compare the performance of the model with other synthetic models using as reference the predictions provided by hourly time series of 217 Class I meteorological stations of TMY3 database.

The comparison shows that the model is very consistent for different tilted planes and provides yearly predictions for final energy yields whose maximum bias and root mean square deviations with respect to TMY 3 data are lower than $\pm 2 \%$ when using Hay model for diffuse sky radiation.

\section{Acknowledgement}

This work has been possible thanks to the funding of the FP7ENERGY European Programme in the project PhotoVoltaic Cost reduction, Reliability, Operational performance, Prediction and Simulation (PVCROPS). Project reference: 308468. 